\title{
Analysis of Enterovirus Genotypes in Cerebrospinal Fluid of Children Associated with Aseptic Meningitis in Liaocheng, China, from 2018 to 2019
}

\section{Jing Wang}

Liaocheng People's Hospital

\section{Min Meng}

Liaocheng People's Hospital

Huan Xu

Liaocheng People's Hospital

Ting Wang ( $\nabla$ tinawang1003@163.com )

Liaocheng People's Hospital https://orcid.org/0000-0001-8683-2956

\section{Ying Liu}

Liaocheng People's Hospital

\section{Han Yan}

Liaocheng People's Hospital

\section{Peiman Liu}

Liaocheng People's Hospital

\section{Daogang Qin}

Liaocheng People's Hospital

\section{Qiaozhi Yang}

Liaocheng People's Hospital

\section{Research article}

Keywords: Enterovirus (EV), Aseptic meningitis, Phylogenetic analysis, Children, Liaocheng

Posted Date: February 19th, 2021

DOl: https://doi.org/10.21203/rs.3.rs-251819/v1

License: (c) (i) This work is licensed under a Creative Commons Attribution 4.0 International License. Read Full License 


\section{Abstract}

Background: Aseptic meningitis is the most common viral infection caused by human enteroviruses, but enteroviruses associated with aseptic meningitis in Liaocheng have not been reported yet. The aim of this study was to determine prevalence and genetic characteristics of enteroviruses causing aseptic meningitis in children in Liaocheng.

Methods: We reviewed the epidemiological and clinical characteristics of 504 pediatric cases with aseptic meningitis in Liaocheng from 2018 to 2019, and analyzed the phylogeny of the predominant enterovirus (EV) types causing this disease.

Results: A total of 107 children were positive for EV by the nested-PCR in cerebrospinal fluid samples. Most of the positive patients were children within 13 years old, had symptoms such as fever, headache and vomiting $(P<0.05)$. The most prevalent seasons of EV-positive cases accurred in summer and autumn. The $107 \mathrm{EV}$ sequences belonged to 8 serotypes, and echovirus types 18,6 and 11 were the three dominant serotypes in Liaocheng during the 2-year period. Phylogentic analyses demonstrated that E18 and $\mathrm{E} 6$ isolates belonged to subgenotype $\mathrm{C} 2$, while E11 isolates belonged to subgenotype D5. The VP1 analysis suggested that only one lineage of these three types were co-circulating in Liaocheng region.

Conclusions: This study demonstrated the diversified enterovirus genotypes attributing to the large outbreak of aseptic meningitis in Liaocheng. Therefore, the large-scale surveillance is required to the epidemiology of enteroviruses associated with aseptic meningitis, and is important for diagnosis and treatment of aseptic meningitis in Liaocheng.

\section{Introduction}

The enteroviruses (EVs) are small, nonenveloped, positive single-stranded RNA viruse of the genus Enterovirus within the family Picornaviridae [1]. To date, 116 serotypes belonging to four enterovirus species (EV-A, B, C, and D) have been identified to infect humans on the basis of neutralizing assays and sequence analysis of the major capsid VP1 gene [2]. EVs are predominantly spread from person to person via the fecal-oral route and infect approximately a billion people worldwide each year, and they occur typically in the summer and autumn in restricted geographical areas or communities, leading to increase admissions to hospital wards for short periods. [3]. They are ubiquitous and cause diverse clinical manifestations, from mild presentations including minor febrile illness, gastrointestinal diseases and rash to more severe syndromes such as acute flaccid paralysis, myocarditis, neonatal sepsis, acute haemorrhagic conjunctivitis, encephalitis and aseptic meningitis [4].

Aseptic meningitis, defined as the most commonly observed CNS infection with sterile cerebrospinal fluid (CSF), causes different clinical presentations according to the patient's age and immune status [5]. Nonpolio human enteroviruses (EVs), especially the species enterovirus $B(E V-B)$, are the most common etiology for this disease [6]. Various studies over the past several decades have shown that most EV-B types are the major causative agents for this disease, and numerous EV-Bs associated meningitis 
outbreaks have been reported throughout the world $[7,8,9,10,11,12,13]$. Shandong is a coastal province in China. There had been outbreaks of aseptic meningitis caused by EV-Bs (e.g., E6, E30, CVB3 and CVB5) in middle east of Shandong Province $[14,15,16,17,18]$. However, Liaocheng city is located in the western region of Shandong province, the information on the EVs causing aseptic meningitis in children has not been reported. There is limited information on circulating enterovirus serotypes and associated clinical phenotypes in pediatric population in Liaocheng region. Hence, such knowledge is essential for laboratory diagnostics, patient management and future outbreak response.

In this study, the main aim was to investigate the genotypes of enterovirus contributing to aseptic meningitis and the associations between enterovirus types and clinical manifestations and CSF laboratory findings in Liaocheng over the period from 2018 to 2019. Furthermore, the intratypical genetic variation of three predominant EV types was carried out through nested RT-PCR and phylogenetic analysis based on VP1 region directly in CSF samples of patients.

\section{Materials And Methods}

\section{Patients and clinical samples collection}

Liaocheng city is located in the western region of Shandong province between $35^{\circ} 47^{\prime} \sim 37^{\circ} 02^{\prime}$ north latitude and $115^{\circ} 16^{\prime} \sim 116^{\circ} 32^{\prime}$ east longitude. Liaocheng covers an area of $8715 \mathrm{~km}^{2}$ accounting for $5.5 \%$ of Shandong province. This area has a population of 5,935,700, with the population density of 681.1 people per square kilometers.

504 cerebrospinal fluid (CSF) specimens from children admitted with suspected aseptic meningitis were collected in Liaocheng People's Hospital (China) from 1 January 2018 to 31 December 2019. CSF specimens were sent to our laboratory at about $4{ }^{\circ} \mathrm{C}$ during sample transport and stored at $-80^{\circ} \mathrm{C}$ for further analysis. Data on demographics, clinical symptoms, and CSF laboratory findings were collected retrospectively from patients' clinical history. All statistical tests were analyzed using SPSS software version 18.0. Data were presented as Mean \pm Standard Error (SE) for continuous variables, and a P-value less than 0.05 was considered statistical differences $(P<0.05)$.

\section{Enterovirus diagnosis and molecular genotyping}

Viral RNA was extracted from $200 \mu$ of the CSF samples by using the MagBeads RNA Extraction Kit (Liferiver, China) according to the manufacturer's recommended procedure. Species-specific nested primers were used to amplify the region of VP1 gene of EV-A and EV-B based on the standard protocol [19].

Reverse transcription polymerase chain reaction (RT-PCR) and PCR were conducted using the SuperScript ${ }^{\mathrm{TM}}$ III OneStep RT-PCR System (Invitrogen, USA) and the Pyrobest ${ }^{\mathrm{TM}}$ DNA Polymerase (TaKaRa, Japan). For the first round, the PCR was performed in a $25 \mu$ reaction containing $8 \mu \mathrm{l}$ RNA, $1 \mu \mathrm{l}$ 
RT-PCR mix, 12.5 ul 2xReaction Buffer, and 20 pmol each of outer primers for EV-A or EV-B. Amplification was as follows: $50{ }^{\circ} \mathrm{C}$ for $30 \mathrm{~min}$, pre-denaturing stage at $94^{\circ} \mathrm{C}$ for $2 \mathrm{~min}$, denaturing stageat $94{ }^{\circ} \mathrm{C}$ for 30 $\mathrm{s}$, annealing stage at $52{ }^{\circ} \mathrm{C}$ for $30 \mathrm{~s}$ and elongating stage at $72{ }^{\circ} \mathrm{C}$ for $1 \mathrm{~min} ; 30$ cycles were performed. Subsequently, the second round PCR was performed with $2 \mu \mathrm{l}$ of the former PCR products and 20 pmol each of inner primers in a volume of $25 \mu$ under the same PCR conditions as described above. The PCR product was harvested by electrophoresis through $1.0 \%$ agarose gel, ethidium bromide staining and UV illumination. The positive PCR products were purified and bi-directionally sequenced by using an ABI3730XL automatic sequencer (Applied Biosystems, FosterCity, CA, USA). The obtained sequences were used for molecular typing by using on line Enterovirus Genotyping Tool version 0.1.

\section{Sequence analysisand phylogenetic analysis}

BLASTn web site was used to align multiple partial VP1 sequences

(https://blast.ncbi.nlm.nih.gov/Blast.cgi). Nucleotide sequence alignment and homologous comparisons were performed using the BioEdit software (version 7.0.5.3). Multiple alignments were generated with the Clustal W program. Phylogenetic analyses based on the VP1 sequences were constructed by in MEGA 6 program using the neighbor-joining $(\mathrm{N} J)$ method with maximum composite likelihood and Kimura 2parameter models [20]. Bootstrap testing with 1000 duplicates was used to test the strength of the phylogenetic trees. Bootstrap values $>70 \%$ were considered statistically significant for grouping. In addition, the nucleotide difference of more than $15 \%$ between groups and $8 \%$ within groups in the VP1 region was used to distinguish genotypes and sub-genotypes.

\section{Nucleotide sequence accession number}

The complete VP1 sequences of the EV strains obtained in this study were deposited in the GenBank database under the accession numbers MT901934-MT901942, MT950542-MT950636, MT646123MT646125.

\section{Results}

\section{Cases and epidemiology}

A total of 504 hospitalized patients suspected of having aseptic meningitis were reported in the period from 2018 to 2019 . Of the patients, 107 (21.2\%) were positive for enteroviruses by RT-PCR. Figure 1 showed the age distribution for all the cases. For EV-positive patients, age ranged from 1 month to 13 years old. The gender ratio was 1.97:1, with 71 male and 36 female cases. There were no significant differences between EV-positive patients and the negative patients in male-to-female ratio $(P>0.05)$. When analyzing for the monthly distribution of patients, enteroviral infections were observed throughout the year, whereas $98.1 \%(105 / 107)$ of the cases predominate in summer and autumn (Fig. 2). The majority of the cases occurred between June and October, and mostly in July. 


\section{Clinical Characteristics And Laboratory Findings}

The clinical characteristics and laboratory findings of EV-positive and EV-negative patients were shown in Table 1. The most common clinical symptoms of EV-positive patients were fever, headache and vomiting. Approximately $94.4 \%$ of the EV-positive patients suffered from fever at the time of hospital admission. There were no significant differences between EV-positive patients and the negative patients in hospital stay and fever duration before admission $(P>0.05)$. The data showed that the frequency of headache, dizzy and neck stiffness was significantly higher in EV-positive patients than in the negative patients $(P<$ 0.05). Almost half the patients had complications such as neurologic, circulatory or respiratory system symptoms. CSF pleocytosis and protein levels were found to be significantly higher in EV-positive group than in the negative group $(P<0.05)$. In general, EV was found to be statistically associated with pleocytosis with $92.5 \%(99 / 107)$ in EV-positive cases, whereas still $7.5 \%(8 / 107)$ of the EV cases did not have pleocytosis. 
Table 1

Comparison of clinical characteristics and laboratory findings between EV-positive patients and EVnegative patients from 2018 to 2019.

\begin{tabular}{|c|c|c|c|}
\hline Characterics & $\begin{array}{l}\text { EV-positive patients } \\
(n=107)\end{array}$ & $\begin{array}{l}\text { EV-negative patients } \\
(n=397)\end{array}$ & $P$ value \\
\hline Age $^{a}$ (years) & $6.6 \pm 3.1$ & $5.6 \pm 3.1$ & 0.019 \\
\hline Male [no. (\%)] & $71(66.4)$ & $258(65)$ & 0.792 \\
\hline Hospital stay ${ }^{a}$ (days) & $9.3 \pm 2.3$ & $9.2 \pm 2.2$ & 0.902 \\
\hline Fever duration before admissiona (days) & $2.3 \pm 2.4$ & $2.9 \pm 2.8$ & 0.059 \\
\hline \multicolumn{4}{|l|}{ Clinical symptoms and signs [no. (\%)] } \\
\hline Fever & 101(94.4) & $366(92.2)$ & 0.438 \\
\hline Headache & 98(91.6) & $304(76.6)$ & 0.001 \\
\hline Vomiting & $47(44)$ & $185(46.6)$ & 0.622 \\
\hline Lethargy & $44(41.1)$ & $129(32.5)$ & 0.095 \\
\hline dizzy & $56(52.3)$ & $148(37.3)$ & 0.005 \\
\hline Convulsion & $1(0.9)$ & $28(7.1)$ & 0.016 \\
\hline Neck stiffness & $19(17.8)$ & $30(7.6)$ & 0.002 \\
\hline Anterior fontanelle bulge & $0(0)$ & $4(1)$ & 0.297 \\
\hline Babinski's sign and/or Brudzinski's sign & $19(17.8)$ & $116(29.2)$ & 0.017 \\
\hline Respiratory symptoms ${ }^{b}$ & 78(72.9) & $239(60.2)$ & 0.017 \\
\hline \multicolumn{4}{|l|}{ CSF findings [no. (\%)] } \\
\hline White blood cell $>10 \times 10^{6} / \mathrm{L}$ & $99(92.5)$ & 275(69.3) & $<0.001$ \\
\hline Protein $>40 \mathrm{mg} / \mathrm{dL}$ & 21(19.6) & $37(9.3)$ & 0.003 \\
\hline a mean \pm SD & & & \\
\hline
\end{tabular}

\section{Enterovirus Genotypes}

Of the 504 specimens, 107 (21.2\%) were positive for enteroviruses by RT-PCR, which were successfully serotyped by sequencing the amplicon of VP1 sequence. A total of 8 serotypes were detected with different detection rates: E6 (15.9\%, 17/107), E18 (45.8\%, 49/107), E11 (11.2\%, 12/107), CVB5 (8.4\%, 
9/107), CVA9 (6.5\%, 7/107), E30 (6.5\%, 7/107), E5(4.7\%, 5/107), and E20 (1\%, 1/107). All of the serotypes were included within the EV-B species. The positive rate of enterovirus was 18.2\% (50/275) in 2018 and $24.9 \%(57 / 229)$ in 2019 . The proportion of predominant pathogen varied significantly between years, as did the proportion of each serotype. The predominant serotypes witched from E6 $(34 \%, 17 / 50)$ in 2018 to E18 $(66.7 \%, 38 / 57)$ in 2019. Genotypes E-18, E-6, and E-11 were the three predominant types throughout the years of 2018 to 2019.

\section{Phylogenetic Analysis Of Enterovirus Genotypes}

Phylogenetic analysis and homologous comparison were conducted using the complete VP1 sequences of $49 \mathrm{E} 18,17 \mathrm{E} 6$ and $12 \mathrm{E} 11$ isolates in this study, and the global reference sequences of $43 \mathrm{E} 18,59 \mathrm{E} 6$ and 75 E11 retrieved from GenBank which are representatives of different genotypes and subgenotypes, respectively.

All Liaocheng E-18 isolates were classified into the C2 subgenotype alongside with the meningitis-related E18 isolates from the countries of China, France, Tunisia, Korea, Thailand and Australia. E18-314, former reference strain causing aseptic meningitis outbreak in Hebei in 2015, also belonged to this subgenotype. Homologous analysis revealed they shared $94.6 \%-96.2 \%$ nucleotide identity with the meningitis-related E18 strain from Hebei Province (Fig. 3a).

Similarly, 17 E6 VP1 sequences from Liaocheng fell into the C2 subtype along with isolates from different areas of China, Poland, Russia and Belarus. Shandong E6 meningitis isolates in 2014, also clustered in this subgenotype. Liaocheng E6 had more close relationship with Shandong meningitis isolates and shared $95.7-96.7 \%$ similarities with each other (Fig. 3b).

Also, all Liaocheng E11 VP1 sequences grouped into the subgenotype D5 along with former Chinese isolates and foreign isolates from Tunisia, New Zeland, the United States, France and Russia. The E-11 strains of meningitis and HFMD (hand, foot, and mouth disease) were also classified into subgenotype D5 in Fujian and Sichuan Province of China in 2011, 2016 and 2017. Liaocheng E11 had 91.1-98.5\% VP1 nucleotide similarities with these reference strain (Fig. 3c).

\section{Discussions}

Aseptic meningitis is the most common CNS infection with cerebrospinal fluid (CSF) negative for bacteria, and EVs, human parechovirus (HPeV), Varicella zoster virus (VZV) and Herpes simplex virus (HSV) 1 and 2 are the most common viral etiologies. [21, 22]. Specifically, EVs are widely recognized as the main causal agent of aseptic meningitis and association is proved by virus genotype from CSF specimen, which occur in both children and adults. [23]. Various studies over several decades have shown children are the primary victims of this disease, and numerous enterovirus meningitis outbreaks associated with EVs have been described $[23,24,25,26]$. Though, the surveillance system of populationbased EVs in mainland China is limited, several related studies of enterovirus meningitis outbreaks have 
been reported frequently in the provinces of Jiangsu [27], Gansu [28], Anhui [29], Zhejiang [30, 31, 32], Guangdong [8, 33, 34], Yunnan [10], Hebei [35] and Shandong [14, 15, 16, 17, 18, 36, 37, 38] in recent years. These outbreaks were characterized by a large number of hospitalized children, which has become a a pressing issue to public health in current China. Hence, rapid EV identification and genotyping from the CSF samples are critical to investigate the EV circulation and understand the social burden of EV infection.

In this study, we described the molecular epidemiology and enterovirus genotypes from CSF samples of children hospitalized for aseptic meningitis in Liaocheng, China. Our results showed that 107 out of 504 (21.2\%) cases of aseptic meningitis were found positive for enteroviruses and 8 different enteroviruses genotypes were responsible for enterovirus meningitis. Several previous studies showed the identification rate of EV types from the CSF ranged from 19.5-54.1\% using previously developed assays [39, 40, 41, 42]. The findings in our study are consistent with these studies and the success rate of direct EV typing was low (21.2\%). This could be attributed to the low viral load of CSF samples, the high variability of the VP1 region or other virus infection.

The majority of EV cases $(53.3 \%, 57 / 107)$ in this study were children aged between three and six, which occurs most frequently in preschool children. Previous studies showed that a higher prevalence of enteroviral meningitis in males vs. females [18, 32]. In our study, the findings are similar with these reports and the male-to-female ratio was found to be $1.97: 1$. In the present study, $105 \mathrm{EV}$ cases $(98.1 \%$, 105/107) were detected in the summer and autumn seasons, which appears with a peak in July. Only 2 cases were detected in spring. Our results revealed that EV infection has typical seasonal feature in temperate climates. This is consistent with other studies showing that most aseptic meningitis occur in summer and autumn, and less frequent in the spring and winter [11, 32].

The clinical signs and symptoms of enteroviral meningitis in children were mostly nonspecific, with fever and headache being the most common symptoms in this study followed by vomiting. Among the 107 patients with enteroviral meningitis, 101 cases $(94.4 \%, 101 / 107)$ suffered from fever at the time of hospital admission, which is in congruence with previous studies [43]. Although the EV-positive patients and the negative patients exhibited different clinical symptoms, these symptoms were not enough to distinguish between different etiologies. CSF WBC and protein levels were elevated in most EV-positive cases in this study, and in comparison to the EV-negative groups, significant differences were observed. Whereas, $8 \mathrm{EV}$ cases did not have pleocytosis, which is in congruence with other previous studies $[44,45$, 46].

In this study, a total of the 8 enterovirus genotypes were detected by sequencing the VP1 region of the EV genome from 2018 to 2019, which were found to belong to EV species B (E18, E6, E11, CVB5, CVA9, E30, E5 and E20). During the 2-year period, the predominant enterovirus types were E18 (45.8\%), E6 (23.8\%) and E11 (20.5\%), and these are associated most commonly with aseptic meningitis. The enterovirus positive rate was $18.2 \%$ in 2018 and $24.9 \%$ in 2019 , and the proportion of predominant genotype varied 
significantly between years. We found that E6, E18, and E11 was the primary causative agent in 2018, while only E18 as the dominant pathogen in 2019.

The phylogenetic analysis of VP1 gene in the present study has been widely used for typing EVs and molecular epidemiological investigations. Some EV serotypes (e.g., E6, E11, E18, E30 and CVB5) are still causing global epidemic outbreaks, while others (e.g., CVA9, E5 and E20) are primarily endemic infections $[18,47,48,49,50,51]$. E18 has caused many aseptic meningitis outbreaks in Germany, the United States, Japan, Korea, and other countries or regions $[50,52,53,54,55,56]$. However, in mainland China, E18 meningitis was less frequently reported. In China, E18 encephalitis/meningitis outbreak was first reported in Hebei Province in 2015 [35]. Subsequently, E18-associated aseptic meningitis occurred in Zhejiang province in 2014 and 2017 [32]. Previous studies showed that all E18 strains segregated into three genotypes: A, B, and C. Genotype $C$ could be further divided into subgenotypes $C 1$ and $C 2$ [35]. Genotypes $A$ and subgenotypes $\mathrm{C} 2$ had been circulated in mainland China. The subgenotype $\mathrm{C} 2 \mathrm{had}$ a wide geographical distribution and was the absolute dominant subgenotype in mainland China in recent five years, whereas genotype A disappeared after 2005. In this study, all Liaocheng E18 isolates belonged to subgenotype $\mathrm{C2}$. Chen et al. reported that the meningitis outbreak in Hebei Province was caused by a new C2 E18 strain, our study supported this observation. Liaocheng E18 had evolutionally close relationship with the reference E18-314 strain circulating in the neighboring Hebei Province, which suggested that E18314 formed an exclusive transmission chain in China. As frequent travel or the returning of migrant workers to Hebei Province might increase importation of E18, continuous surveillance is needed.

In China, E6 was first reported to be associated with aseptic meningitis outbreak in 2005 [29]. Then Zhejiang province has also detected E6 meningitis outbreak in 2014 and 2017 [32]. In Shandong, sporadic E6 cases were occasionally observed, and environmental surveillances on sewage had proved that $E 6$ was the predominant serotype in certain years $[15,16,57,58]$. Furthermore, E6 was one of the predominant types that are responsible for enterovirus meningitis in 2014 [18]. Previous studies showed that all known E6 strains divided into three genotypes-A, B and C. Genotype C could be subdivided into four subgenotypes $C 1$ to $C 4$ [29]. Phylogenetic analyses showed that the subgenotype $C 2$ became the most frequently detected subgenotype in mainland China after 2014, while subgenotype C4 appeared extinct after 2005. Liaocheng E6 isolates belonged to C2 subgenotype, which was most closely related to the meningitis strains isolated in 2014 in Shandong province, suggesting far less variety and continuous circulation in Shandong province.

Echovirus 11 (E11) is one of the most common cause of meningitis in Russia, America, India, Japan and Israel, but the meningitis outbreak of E11 has not been found in mainland China [59, 60, 61, 62]. Though in China, no E11 meningitis outbreak has been reported, EV surveillance has been performed, and E11 cases with meningitis, AFP (acute flaccid paralysis) and HFMD were observed on sewage or CSF conducted in provinces of Shandong, Fujian, Yunnan, and so on $[48,63,64,65]$. In this study, phylogenetic analysis revealed that Liaocheng E11 grouped into subgenotype D5. In previous epidemiology study on worldwide E11, genotype A was the predominant genotype in mainland China, and D5 was the predominant subgenotype circulated in American, European and Russia [66]. However, E11 
meningitis caused by subgenotype D5 was reported in Fujian Province in China in 2011, and E11 isolated from the HFMD surveillance system in China in 2016-2017 were also particularly classified into subgenotype D5 $[48,65]$. In this study, no Liaocheng E11 belonged to genogroup A, and a great number of subgenotype D5 were identified, suggesting meningitis-related E11 circulating in China is mainly composed of isolates of this subgenotype in recent years.

There are some limitations to this study. Firstly, the study was conducted in a tertiary hospital and the number of cases was low. Secondly, the findings are limited by the use of nest RT-PCR performed for the detection of EVs in CSF samples, the viral load of the samples was low and other virus infection has not been involved in our study. In the future, to elucidate further the epidemiology of the pathogens for aseptic meningitis, a prospective multicentric larger studies will be developed and other pathogens such as HPeV, VZV, HSV-1 and HSV-2 should be taken and evaluated.

\section{Conclusions}

Our study describes that EVs are the common etiology of aseptic meningitis among children in Liaocheng, China. Furthermore, this study reveals that eight meningitis-related EV genotypes circulate in Liaocheng. E18, E6 and E11 are the three predominant genotypes. The subgenotype of E18 and E6 are C2, and the subgenotype of E11 is D5. Phylogenetic analyses of these three types exhibit one lineage circulating in Liaocheng region and they all have great genetic diversity with foreign isolates. Further surveillance of EV genotypes should be needed to understand the changing genetic characteristics and clinical pathogenicity, and optimize the choice of interventions.

\section{Abbreviations}

CSF: Cerebrospinal fluid; EV: Enterovirus; E5: Echovirus 5; E6: Echovirus 6; E11: Echovirus 11; E18: Echovirus 18; E20: Echovirus 20; E30: Echovirus 30; CVA9: Coxsackie virus A9; CVB5: Coxsackie virus B5; RT-PCR: Reverse transcription polymerase chain reaction; VP1: Viral capsid protein 1; HPeV: human parechovirus; VZV: Varicella zoster virus; HSV1: Herpes simplex virus 1; HSV2: Herpes simplex virus 2; HFMD: hand, foot, and mouth disease; AFP: acute flaccid paralysis.

\section{Declarations}

\section{Ethics statement}

This study was approved by the Ethics Committee at Liaocheng Municipal Center for Disease Control and Prevention, China. Written informed consent was obtained from the parents of every child participant enrolled in this study.

\section{Consent for publication}


Not applicable.

\section{Competing interests}

All authors declare that they have no competing financial interests.

\section{Funding}

This work was supported by the Natural Science Foundation of Shandong Province (ZR2017PH054), Shandong Traditional Chinese Medicine Development Project (2017494), Shandong Province Medical and Health Development Plan Project (2018WS427), and Shandong Traditional Chinese Medicine Science and Technology Development Plan Project (2019-0885).

Author Contributions

JW: data collection, analysis and interpretation, laboratory testing, drafting, review and edit of the manuscript, funding acquisition. MM: conception and study design, data collection and analysis, drafting, laboratory testing, review and edit of the manuscript. HX: data collection, laboratory testing. TW: conception and study design, review and edit of the manuscript. HY and YL: data collection. PML, DGQ, QZY: conception and study design. All authors read and approved the final manuscript.

Acknowledgments

Not applicable.

\section{References}

1. Pons-Salort M, Parker EP, Grassly NC. The epidemiology of non-polio enteroviruses: recent advances and outstanding questions. Curr Opin Infect Dis. 2015;28:479-87.

2. Walker PJ, Siddell SG, Lefkowitz EJ, Mushegian AR, Dempsey DM, Dutilh BE, et al. Changes to virus taxonomy and the International Code of Virus Classification and Nomenclature ratified by the International Committee on Taxonomy of Viruses (2019). Arch Virol. 2019;164(9):2417-29.

3. Jubelt B, Lipton HL. Enterovirus/Picornavirus infections. Handb Clin Neurol. 2014;123:379-416.

4. Nikonov OS, Chernykh ES, Garber MB, Nikonova EY. Enteroviruses: classification, diseases they cause, and approaches to development of antiviral drugs. Biochemistry. 2017;82(13):1615-31.

5. Martin NG, Iro MA, Sadarangani M, Goldacre R, Pollard AJ, Goldacre MJ. Hospital admissions for viral meningitis in children in England over five decades: a population-based observational study. Lancet Infect Dis. 2016;16(11):1279-87.

6. Tattevin P, Tchamgoué S, Belem A, Bénézit F, Pronier C, Revest M. Aseptic meningitis. Rev Neurol (Paris). 2019;175(7-8):475-80. 
7. Oberste MS, Maher K, Kennett ML, Campbell JJ, Carpenter MS, Schnurr D, et al. Molecular epidemiology and genetic diversity of echovirus type 30 (E30): genotypes correlate with temporal dynamics of E30 isolation. J Clin Microbiol. 1999;37(12):3928-33.

8. Xiao H, Guan D, Chen R, Chen P, Monagin C, Li W, et al. Molecular characterization of echovirus 30associated outbreak of aseptic meningitis in Guangdong in 2012. Virol J. 2013;10:263.

9. Holmes CW, Koo SS, Osman H, Wilson S, Xerry J, Gallimore Cl, et al. Predominance of enterovirus B and echovirus 30 as cause of viral meningitis in a UK population. J Clin Virol. 2016;81:90-3.

10. Zhu Y, Zhou X, Liu J, Xia L, Pan Y, Chen J, et al. Molecular identification of human enteroviruses associated with aseptic meningitis in Yunnan province, Southwest China. Springerplus. 2016;5(1):1515.

11. Smuts H, Cronje S, Thomas J, Brink D, Korsman S, Hardie D. Molecular characterization of an outbreak of enterovirus-associated meningitis in Mossel Bay, South Africa, December 2015-January 2016. BMC Infect Dis. 2018;18(1):709.

12. Miyoshi M, Goto A, Komagome R, Yamaguchi H, Maruo Y, Nakanishi M, et al. Genetic characterization of a novel recombinant echovirus 30 strain causing a regional epidemic of aseptic meningitis in Hokkaido, Japan, 2017. Arch Virol. 2020;165(2):433-8.

13. Ramalho E, Sousa I Jr, Burlandy F, Costa E, Dias A, Serrano R, et al. Identification and phylogenetic characterization of human enteroviruses isolated from cases of aseptic meningitis in Brazil, 20132017. Viruses. 2019;11(8):690.

14. Wang HY, Xu AQ, Zhu Z, Li Y, Ji F, Zhang Y, et al. The genetic characterization and molecular evolution of echovirus $\mathbf{3 0}$ during outbreaks of aseptic meningitis. Zhonghua Liu Xing Bing Xue Za Zhi. 2006;27(9):793-7.

15. Tao Z, Song Y, Wang H, Zhang Y, Yoshida H, Ji S, et al. Intercity spread of echovirus 6 in Shandong Province, China: application of environmental surveillance in tracing circulating enteroviruses. Appl Environ Microbiol. 2012;78(19):6946-53.

16. Tao Z, Song Y, Li Y, Liu Y, Jiang P, Lin X, et al. Coxsackievirus B3, Shandong Province, China, 19902010. Emerg Infect Dis. 2012;18(11):1865-7.

17. Chen P, Tao Z, Song Y, Liu G, Wang H, Liu Y, et al. A coxsackievirus B5-associated aseptic meningitis outbreak in Shandong Province, China in 2009. J Med Virol. 2013;85(3):483-9.

18. Chen $P$, Lin $X$, Liu G, Wang S, Song L, Tao Z, et al. Analysis of enterovirus types in patients with symptoms of aseptic meningitis in 2014 in Shandong, China. Virology. 2018;516:196-201.

19. Leitch EC, Harvala H, Robertson I, Ubillos I, Templeton K, Simmonds P. Direct identification of human enterovirus serotypes in cerebrospinal fluid by amplification and sequencing of the VP1 region. $J$ Clin Virol. 2009 Feb;44(2):119-24.

20. Stecher G, Liu L, Sanderford M, Peterson D, Tamura K, Kumar S. MEGA-MD: molecular evolutionary genetics analysis software with mutational diagnosis of amino acid variation. Bioinformatics. 2014;30:1305-7.

21. Putz K, Hayani K, Zar FA. Meningitis. Prim Care. 2013;40(3):707-26. 
22. Wright WF, Pinto CN, Palisoc K, Baghli S. Viral (aseptic) meningitis: A review. J Neurol Sci. 2019;398:176-83.

23. Rudolph $\mathrm{H}$, Schroten $\mathrm{H}$, Tenenbaum T. Enterovirus Infections of the Central Nervous System in Children: An Update. Pediatr Infect Dis J. 2016;35(5):567-9.

24. Zakhour R, Aguilera E, Hasbun R, Wootton SH. Risk classification for enteroviral infection in children with meningitis and negative gram stain. Pediatr Emerg Care. 2018;34(11):791-6.

25. Ai J, Xie Z, Liu G, Chen Z, Yang Y, Li Y, Chen J, Zheng G, Shen K, et al. Etiology and prognosis of acute viral encephalitis and meningitis in Chinese children: a multicentre prospective study. BMC Infect Dis. 2017;17(1):494.

26. Hasbun R, Wootton SH, Rosenthal N, Balada-Llasat JM, Chung J, Duff S, et al. Epidemiology of Meningitis and Encephalitis in Infants and Children in the United States, 2011-2014. Pediatr Infect Dis J. 2019;38(1):37-41.

27. Zhao YN, Jiang QW, Jiang RJ, Chen L, Perlin DS. Echovirus 30, Jiangsu Province, China. Emerg Infect Dis. 2005;11(4):562-7.

28. Cui A, Yu D, Zhu Z, Meng L, Li H, Liu J, et al. An outbreak of aseptic meningitis caused by coxsackievirus A9 in Gansu, the People's Republic of China. Virol J. 2010;7:72.

29. Mao N, Zhao L, Zhu Z, Chen X, Zhou S, Zhang Y, et al. An aseptic meningitis outbreak caused by echovirus 6 in Anhui province, China. J Med Virol. 2010;82(3):441-5.

30. Yan JY, Lu YY, Xu CP, Yu Z, Gong LM, Chen Y, Zhang YJ. Study on the etiological and molecular characteristics of aseptic meningitis epidemic in Zhejiang Province in 2002-2004. Bing Du Xue Bao. 2011;27(5):462-8.

31. Zheng S, Ye H, Yan J, Xie G, Cui D, Yu F, et al. Laboratory diagnosis and genetic analysis of a family clustering outbreak of aseptic meningitis due to echovirus 30. Pathog Glob Health. 2016;110(6):233-7.

32. Sun Y, Miao Z, Yan J, Gong L, Chen Y, Chen Y, et al. Sero-molecular epidemiology of enterovirusassociated encephalitis in Zhejiang Province, China, from 2014 to 2017. Int $J$ Infect Dis. 2019;79:58-64.

33. Xiao H, Huang K, Li L, Wu X, Zheng L, Wan C, Zhao W, Ke C, Zhang B. Complete genome sequence analysis of human echovirus 30 isolated during a large outbreak in Guangdong Province of China, in 2012. Arch Virol. 2014;159(2):379-83.

34. Lu J, Zheng H, Guo X, Zhang Y, Li H, Liu L, et al. Elucidation of echovirus 30's origin and transmission during the 2012 aseptic meningitis outbreak in Guangdong, China, through continuing environmental surveillance. Appl Environ Microbiol. 2015;81(7):2311-9.

35. Chen X, Li J, Guo J, Xu W, Sun S, Xie Z. An outbreak of echovirus 18 encephalitis/meningitis in children in Hebei Province, China, 2015. Emerg Microbes Infect. 2017;6(6):e54.

36. Wang HY, Li Y, Xu AQ, Zhang Y, Tao ZX, Liu GF, et al. Identification and phylogenic analysis of Coxsackie-virus B5 during an outbreak of aseptic meningitis in Shandong. Zhonghua Liu Xing Bing Xue Za Zhi. 2010;31(1):64-8. 
37. Wan YZ, Yue YY, Li P, Li ZH, Li J, et al. Molecula-biological identification of pathogens which caused an outbreak of viral encephalitis in Jinan area. Zhonghua Shi Yan He Lin Chuang Bing Du Xue Za Zhi. 2007;21(4):313-5.

38. Fan QY, Zhou XL, Yang H, Tao ZX, Li Y, Liu GF, et al. Molecular characterization of coxsackievirus B3 isolated from an outbreak of aseptic meningitis in Shandong Province, China. Zhonghua Shi Yan He Lin Chuang Bing Du Xue Za Zhi. 2009;23(6):455-7.

39. Mirand A, Henquell C, Archimbaud C, Chambon M, Charbonne F, Peigue-Lafeuille H, et al. Prospective identification of enteroviruses involved in meningitis in 2006 through direct genotyping in cerebrospinal fluid. J Clin Microbiol. 2008;46(1):87-96.

40. Kadambari S, Bukasa A, Okike IO, Pebody R, Brown D, Gallimore C, et al. Enterovirus infections in England and Wales, 2000-2011: the impact of increased molecular diagnostics. Clin Microbiol Infect. 2014;20(12):1289-96.

41. Krasota A, Loginovskih N, Ivanova O, Lipskaya G. Direct Identification of Enteroviruses in Cerebrospinal Fluid of Patients with Suspected Meningitis by Nested PCR Amplification. Viruses. 2016;8(1):10.

42. Dumaidi $\mathrm{K}$, Al-Jawabreh A. Molecular detection and genotyping of enteroviruses from CSF samples of patients with suspected sepsis-like illness and/or aseptic meningitis from 2012 to 2015 in West Bank, Palestine. PLoS One. 2017;12(2):e0172357.

43. Anahita I, Ali Akbar R, Yousef M, Sayed Yousef M. Prevalence of Enterovirus Meningitis in Children: Report from a Tertiary Center. Maedica (Bucur). 2018;13(3):213-6.

44. Seiden JA, Zorc JJ, Hodinka RL, Shah SS. Lack of cerebrospinal fluid pleocytosis in young infants with enterovirus infections of the central nervous system. Pediatr Emerg Care. 2010;26(2):77-81.

45. Yun KW, Choi EH, Cheon DS, Lee J, Choi CW, Hwang $\mathrm{H}$, et al. Enteroviral meningitis without pleocytosis in children. Arch Dis Child. 2012;97(10):874-8.

46. Ghabouli Shahroodi MJ, Ghazvini K, Sadeghi R, Sasan MS. Enteroviral Meningitis in Neonates and Children of Mashhad, Iran. Jundishapur J Microbiol. 2016;9(5):e19955.

47. Fernandez-Garcia MD, Kebe 0 , Fall AD, Ndiaye K. Identification and molecular characterization of non-polio enteroviruses from children with acute flaccid paralysis in West Africa, 2013-2014. Sci Rep. 2017;7(1):3808.

48. Li J, Yan D, Chen L, Zhang Y, Song Y, Zhu S, et al. Multiple genotypes of Echovirus 11 circulated in mainland China between 1994 and 2017. Sci Rep. 2019;9(1):10583.

49. Lema C, Torres C, Van der Sanden S, Cisterna D, Freire MC, Gómez RM. Global phylodynamics of Echovirus 30 revealed differential behavior among viral lineages. Virology. 2019;531:79-92.

50. Rodà D, Pérez-Martínez E, Cabrerizo M, Trallero G, Martínez-Planas A, Luaces C, et al. Clinical characteristics and molecular epidemiology of enterovirus infection in infants $<3$ months in a referral paediatric hospital of Barcelona. Eur J Pediatr. 2015;174(11):1549-53.

51. Faleye TOC, Adewumi OM, Klapsa D, Majumdar M, Martin J, Adeniji JA. Genome sequences of two dual-serotype-specific echovirus 20 strains from nigeria. Microbiol Resour Announc. 
2019;8(43):e00894-19.

52. Turabelidze G, Lin M, Butler C, Fick F, Russo T. Outbreak of echovirus 18 meningitis in a rural Missouri community. Mo Med. 2009;106(6):420-4.

53. Krumbholz A, Egerer R, Braun H, Schmidtke M, Rimek D, Kroh C. et al. Analysis of an echovirus 18 outbreak in Thuringia, Germany: insights into the molecular epidemiology and evolution of several enterovirus species B members. 2016;205(5):471 - 83.

54. Kusuhara K, Saito M, Sasaki Y, Hikino S, Taguchi T, Suita S. et al. An echovirus type 18 outbreak in a neonatal intensive care unit. Eur J Pediatr. 2008;167(5):587-9.

55. Park K, Yeo S, Baek K, Cheon D, Choi Y, Park J, Lee S. Molecular characterization and antiviral activity test of common drugs against echovirus 18 isolated in Korea. Virol J. 2011;8:516.

56. Tsai HP, Huang SW, Wu FL, Kuo PH, Wang SM, Liu CC, Su IJ, Wang JR. An echovirus 18-associated outbreak of aseptic meningitis in Taiwan: epidemiology and diagnostic and genetic aspects. J Med Microbiol. 2011;60(9):1360-5.

57. Tao Z, Wang H, Li Y, Xu A, Zhang Y, Song L, et al. Cocirculation of two transmission lineages of echovirus 6 in Jinan, China, as revealed by environmental surveillance and sequence analysis. Appl Environ Microbiol. 2011;77:786-3792.

58. Tao Z, Wang H, Li Y, Liu G, Xu A, Lin X, et al. Molecular epidemiology of human enterovirus associated with aseptic meningitis in Shandong Province, China, 2006-2012. PloS One. 2014;9:e89766.

59. Miller DG, Gabrielson MO, Bart KJ, Opton EM, Horstmann DM. An epidemic of aseptic meningitis, primarily among infants, caused by echovirus 11-prime. Pediatrics. 1968;41(1):77-90.

60. Patel JR, Daniel J, Mathan VI. An epidemic of acute diarrhoea in rural southern India associated with echovirus type 11 infection. J Hyg (Lond). 1985;95(2):483-92.

61. Miwa C, Sawatari S. Epidemic of echo 11 virus infection in Gifu Prefecture in 1993. Kansenshogaku Zasshi. 1994;68(10):1251-5.

62. Somekh E, Shohat T, Handsher R, Serour F. An outbreak of echovirus 11 in a children's home. Epidemiol Infect. 2001;126(3):441-4.

63. Yang J, Cui N, Wang H, Tao Z, Liu Y, Zhang H, et al. Evaluating the prevalence and molecular epidemiology of echovirus 11 isolated from sewage in Shandong Province, China in 2010. Virus Genes. 2012;44(3):388-94.

64. Li WF, Tao ZX, Yang J, Lin XJ, Liu Y, Ji F, et al. Temporal fluctuation and genetic characterization of echovirus 11 isolates upon surveillance of environmental sewage in Shandong Province, China. Bing Du Xue Bao. 2014;30(5):561-6.

65. Chen Q, Cao C, Zhang Y, He C, Luo Z, He Y, et al. Genetic analysis of Echovirus 11 isolated from patients with viral encephalitis in Longyan, China. Bing Du Xue Bao. 2015;31(1):36-41.

66. Yarmolskaya MS, Shumilina EY, Ivanova OE, Drexler JF, Lukashev AN. Molecular epidemiology of echoviruses 11 and 30 in Russia: different properties of genotypes within an enterovirus serotype. 
Infect Genet Evol. 2015;30:244-248.

Figures

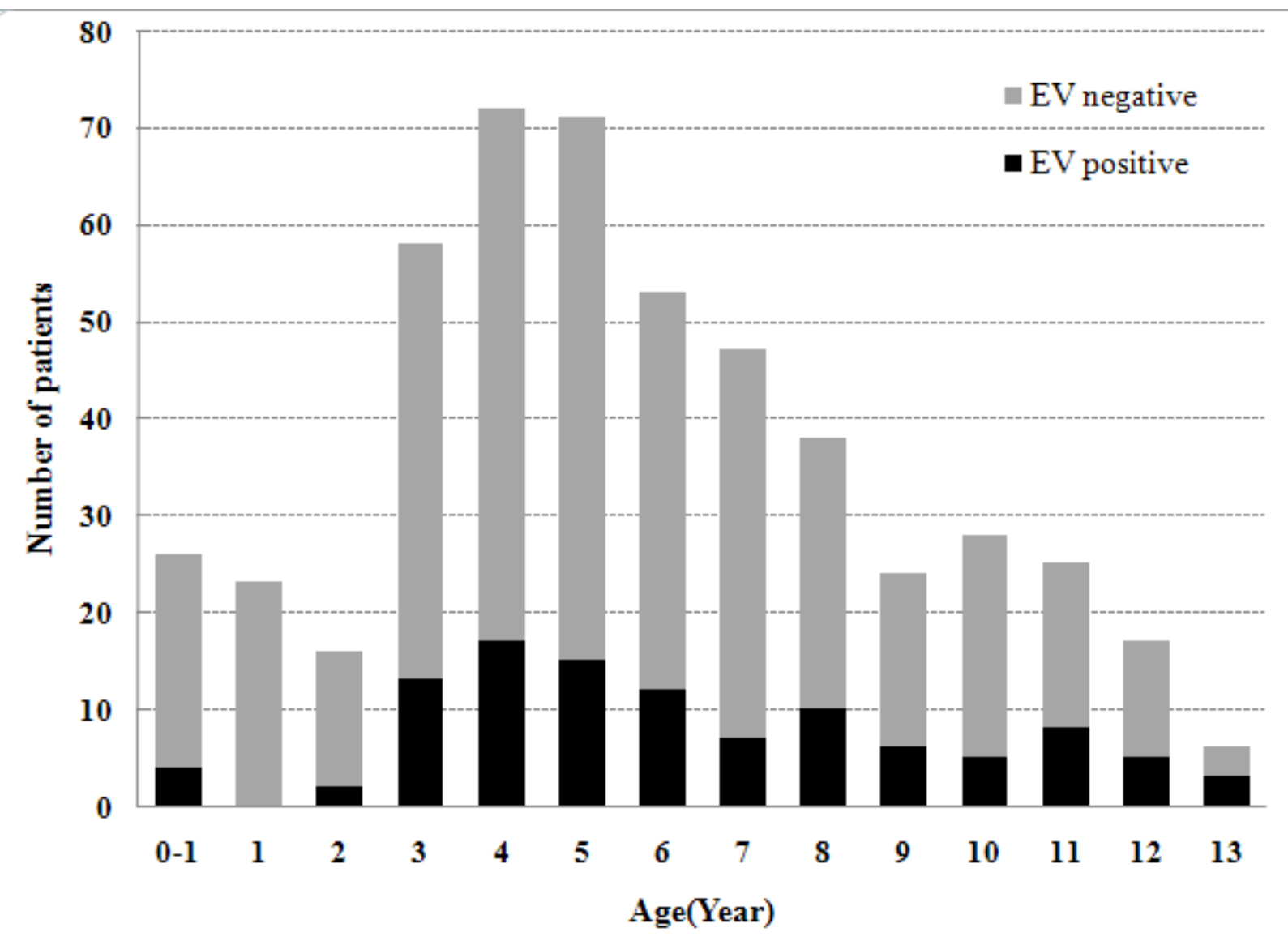

Figure 1

Age distribution of aseptic meningitis patients in Liaocheng $(n=504)$. 


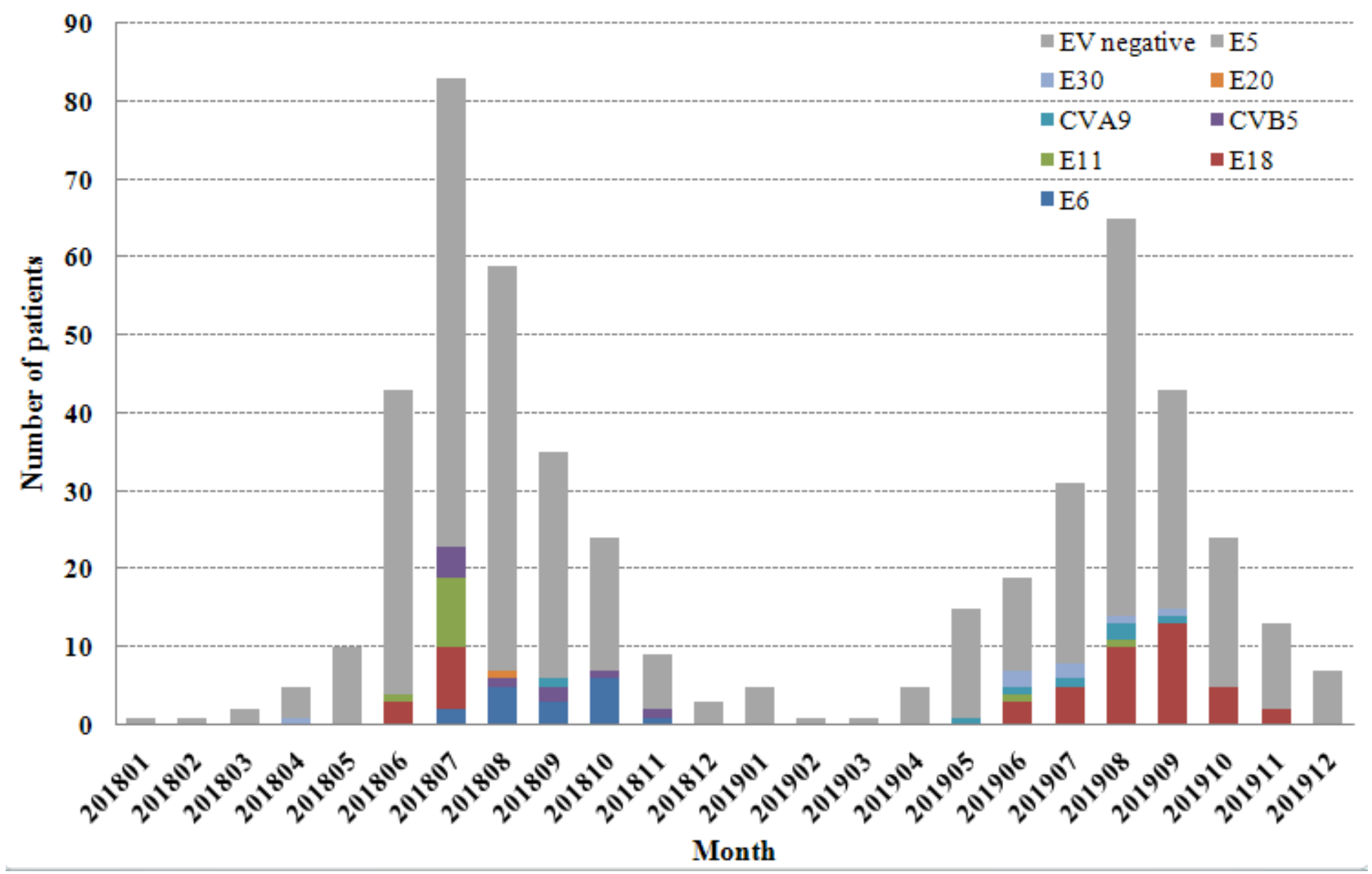

Figure 2

Monthly distribution of enterovirus genotypes identified in aseptic meningitis patients from 2018 to 2019, Liaocheng, China. 


\section{Fig.3}
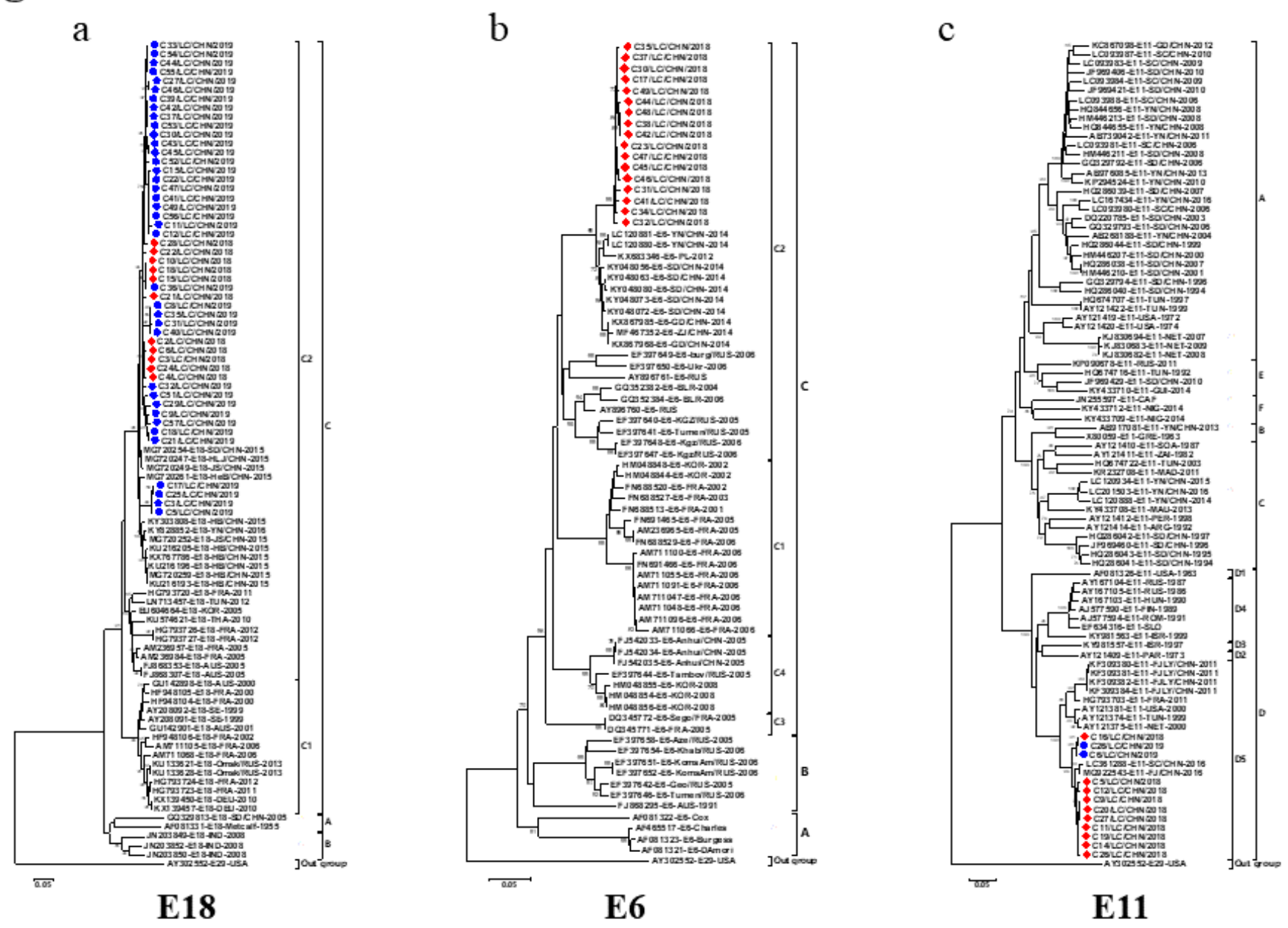

Figure 3

Phylogenetic analyses based on VP1 sequences of E18 (panel a), E6 (panel b) and E11(panel c) causing aseptic meningitis in Liaocheng. Phylogenetic trees are constructed on the partial VP1 gene using Neighbor Joining methods. Red diamond and blue round indicate isolates obtained in this study. The prototype E29-USA strain was used as an outgroup. 